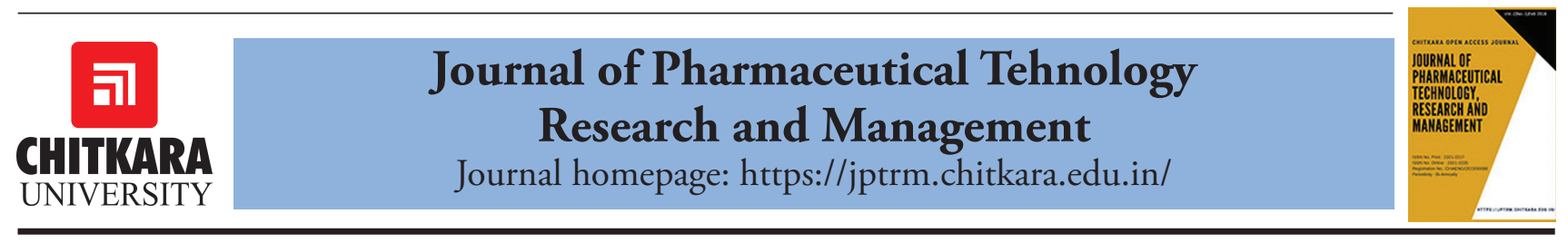

\title{
A Precise Review on Tenofovir Disoproxil Fumarate: An Analytical Profile
}

Vinod A Chaure', Saurabh B Ganorkar ${ }^{1}$, Ganesh U Chaturbhuj², Sanjay J Surana ${ }^{1}$ and Atul A Shirkhedkar ${ }^{1}$

${ }^{1}$ R. C. Patel, Institute of Pharmaceutical Education and Research, Shirpur, Dist: Dhule (MS) India 425405

${ }^{2}$ Institute of Chemical Technology, ICT, Matunga, Mumbai 400019

*Email: shirkhedkar@gmail.com

\section{ARTICLE INFORMATION}

Received: July 11, 2018

Revised: Sep. 17, 2018

Accepted: Nov. 01, 2018

Published online: Nov 02, 2018

Keywords:

Tenofovir Disoproxil Fumarate; Review

Article; Validation; HPLC; HPTLC

\section{ABSTRACT}

Tenofovir Disoproxil Fumarate (TDF) is antiretroviral medicine used treat AIDS as well as chronic Hepatitis-B. TDF is a prodrug of tenofovir and exists as dominant form due to lesser oral bioavailability of parent drug. TDF is now available in a fixed-dose combination with various antiretrovirals like Cobicistat, Efavirenz, Elvitegravir, Emtricitabine, Lamivudine, Rilpivirine, and Nevirapine. Hence, pharmaceutical analysis of TDF and applicability of different analytical methods have gained crucial importance. The present review article assesses the published analytical methods and a variety of approach for investigation of TDF in bulk drug as well as pharmaceutical formulations including combinations. This detailed review includes examination of around eighty analytical methods published during 2008 to 2016 using various techniques which include HPLC, HPTLC, and UV/ Visible-Spectrophotometry. The review also illustrates the scope and limitations of many published analytical methods for analysis of TDF. Such detailed review will be of great help to the researcher who is working on TDF. Miscellaneous methods of rare but unique pharmaceutical distinction have also been given due consideration. The diagrammatic illustrations provide the statistical overview about the various methods referred for analysis of TDF.
The present review offers a critical account on analytical methods published during 2008 to 2016 for determination of TDF

Tenofovir Disoproxil Fumarate (TDF) Fig. 1 is an antiretroviral medicine used to treat HIV/AIDS and chronic hepatitis B (Goicoechea et al., 2008). TDF is a prodrug of Tenofovir (TNF) and exists as dominant form due to low oral bioavailability of TNF. The active substance TNF inhibits the Nucleotide Reverse Transcriptase. TDF is quickly hydrolysed into Tenofovir monophosphate in the body and gets converted into the active drug. The chemical reaction for this conversion in vivo is as represented in Fig. 2(Avihingsanon et al., 2015). TDF is available in the market as tablets; alone and in combination with other drugs. TDF is mostly expelled with Glomerular filtration and in that being transported into renal proximal tubule cells through organic anion transporter-1 (OAT-1). TDF usually considered as a safe drug, but renal toxicities are reported with its use. The reports are available which provides cause of proximal tubulopathy of kidney, Fanconi syndrome, kidney related other toxicities including insipidus calcium and phosphorus dysregulation with bone disease and reduction in Glomerular function (Patel et al., 2010). Besides the aforementioned effects, the antiretroviral therapy (ART) has transformed HIV infection into a manageable, lifelong disease. The first line regimens are critical to successful ART for its long-term treatment (Bygrave et al., 2011).<smiles>CC(C)OC(=O)OCOP(=O)(CO[C@@H](C)CN1CNc2c(N)ncnc21)OCOC(=O)OC(C)C</smiles><smiles>C[C@H](CN1CNc2c(N)ncnc21)OP(=O)(O)O</smiles>

TNF<smiles>CC(C)[C+]C=O</smiles>

Figure 1: Chemical structure of TDF and its metabolism into TNF.

\subsection{Chemistry of Tenofovir Disoproxil Fumarate}

(TDF) is chemically 9-( $R$ )-2-[bis (isopropoxycarbonyl) oxy] methyl] phosphinyl] methoxy] propyl] adenine Fumarate. The molecular formula is $\mathrm{C}_{19} \mathrm{H}_{32} \mathrm{~N}_{5} \mathrm{O}_{10} \mathrm{P}$ and molecular weight is 521.46 . Melting point is $279^{\circ} \mathrm{C}$, drug is faintly soluble in water, soluble in methanol, very slightly soluble in dichloromethane [5]. 


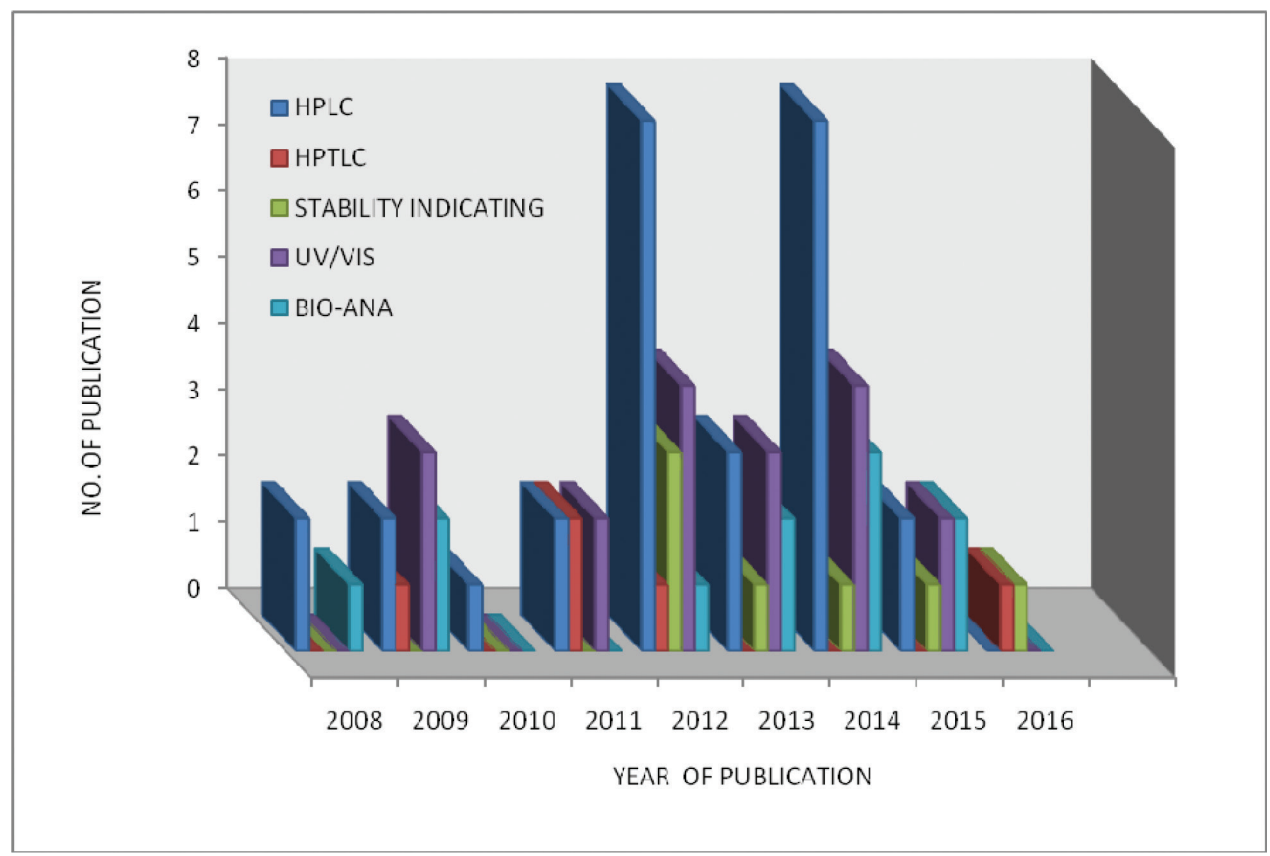

Figure 2: Method Prevalence for Pharmaceutical Determination of TDF.

TDF is absorbed in the gut and quickly converted into TNF. After administration of TDF in the body, ester hydrolysis takes place and in the blood dissociation of two ester linkages occurs. The first cleavage of the ester group offers monoester of TDF then second group of ester gets TNF metabolized intracellularly and followed by its conversion into an anabolite; tenofovir diphosphate which inhibits HIV-1 reverse-transcriptase competitively and stops the DNA synthesis (Kearney et al., 2006).

\section{Pharmacological Profile}

\subsection{Mechanism of Action}

This drug inhibits HIV-1 transcriptase and HBV retrotranscriptase. Tenofovir diphosphate is a weak inhibitor of DNA polymerases $\alpha$ and $\beta$ (mammalian) and DNA polymerase $\gamma$ (mitochondrial) (James et al., 2004).

\subsection{Dosage Forms and Recommended Dose}

TNF is available only in oral tablet dosage forms having $245 \mathrm{mg}$ of TNF corresponding to $300 \mathrm{mg}$ of TDF. The recommended dose for the drug is a tablet per day (James et al., 2004).

\subsection{Pharmacodynamics and Pharmacokinetics}

The range for oral bioavailability is in-between $25 \%$ and 40 $\%$ (depending on the amount of fat), and can be administered safely to patients having liver function impaired (Birkus et al., 2002; Cihlar et al., 2002).

\subsection{Adverse Effects and Contraindications}

Obesity and prolonged nucleoside therapy may be predisposing factors (Porche, 2002). Slight rise in serum creatinine and decrease in serum phosphate levels occurring 4-12 months after starting TDF is characterized by nephrotoxicity (Fontana, 2009).

\section{Various Approaches for Pharmaceutical Analysis}

The use of analytical methods for pharmaceutical analysis is an essential part of drug development and validation procedure. Analytical techniques like UV/VisibleSpectrophotometry, Atomic Absorption, CapillaryElectrophoresis, Liquid -Chromatography either alone or coupled with Mass Spectroscopy, Measurement of Luminescence, Voltammetry and Polarography have been explored for analysis of drugs in bulk as well as in various formulations and biologicals (Rajput et al., 2015). Amongst all these methods, Chromatographic methods HPLC, HPTLC and UV/Visible-Spectrophotometry have generally been studied and preferred over other methods due to sensitivity and simplicity of analysis (Siddiqui et al., 2013).

TDF is available as individual or in combination with other drugs such Emtricitabine, Rilpivirine, Lamivudine, Nevirapine, Efavirenz, Elvitegravir and Cobicistat in various dosage forms.

Thorough literature survey revealed various analytical methods viz HPLC, HPTLC (simple and stability- 
indicating), UV-Spectrophotometry, bio-analytical methods for analysis in human plasma and biological fluids used for analysis of TDF alone or in combination with other drugs. Few pharmaceutical analytical methods such as Capillary-Electrophoresis and specified Electro-Analytical determinations are also critically been reviewed.

\subsection{Chromatographic Analyses}

\subsubsection{HPLC analysis}

Total twenty seven methods have been reported for determination for TDF alone and or in combined dosage form. Reversed-Phase HPLC analyses for TDF (Sarala et al., 2014; SM and Nandedkar, 2009; Sundar and Edla, 2011; Sharma et al., 2012; Balaji et al., 2012; Kandagal et al., 2008; Sharma et al., 2010) Seven RP-HPLC procedures have been reported in literature for determination of TDF using isocratic mode. The identification and separation of the TDF was achieved using C18 column with dimension 250/150 x 4.6 $\mathrm{mm} ; 5 \mu \mathrm{m}$ and variable combination of mobile phases containing acetonitrile, Methanol, and Water. Whereas, in three developed methods combination of Acetontrile/ Methanol and Sodium Hydrogen phosphate, $\mathrm{pH}$ adjusted to 2.3 was chosen as mobile phase. UV/Visible detector / Photo-Diode-Array detectors were used to perform the detection. The retention time for all these methods reported to be below $10 \mathrm{~min}$. Small values of LOD and LOD in the reported methods indicate that these methods are sensitive. All these established methods are claimed to be simple, economical and less time consuming. In many studied analytical methods, the percentage amount of TDF estimated in pharmaceutical formulation was reported to be in the range of $97-101$, indicating superior conformity with the label claimed.

Reversed-Phase HPLC analyses for TDF Combinations (Sharma et al., 2010; Abdelhay et al., 2013; Lavanya et al., 2012; Karunakaran et al., 2010; Srinivasan et al., 2014; Venkatesh et al., 2013; Viswanath et al., 2013; Yenumula et al., 2015; Sharma and Gupta, 2009; Vanitha et al., 2014; Devanaboyina et al., 2012; Kumar et al., 2012; Gorja and Bandla, 2011; Mali et al., 2015; Khan et al., 2014; Jayapalu et al., 2014; Bhavsar et al., 2012; Raju et al., 2008; Ramaswamy Dhas, 2014; Prathap and Rao, 2014; Bhargavi et al., 2012). Total twenty RP-HPLC methods have been reported for analysis of TDF in combination with other drugs. RP-HPLC methods using Isocratic modes have been explored in around sixteen methods; whereas, only four methods were studied using gradient mode of HPLC. In most of these analytical methods C18 column was exclusively applied for separation of TDF and other drugs in its combination. The mobile phase chosen for analysis contains various proportions of Acetonitrile/Methanol and Sodium Hydrogen Phosphate buffer/ water and the $\mathrm{pH}$ adjusted below 7.0. The retention time below $11 \mathrm{~min}$ was reported for TDF and its combinations with other drugs. The coefficient correction value for linearity of both these drugs were reported to be greater than 0.99 .

The detailed account of mobile phases used for determination, sample matrix, wavelength maximum, linearity, retention time and the correlation coefficient is described in Table 1. Whereas, the comparison of the column(s) with the specification(s) for HPLC analysis along with the conditions of flow rate, temperature, detector and type of analysis are quoted in Table 2.

Table 1: HPLC analysis of TDF alone and in its combination with other drugs.

\begin{tabular}{|c|c|c|c|c|c|c|c|}
\hline $\begin{array}{l}\text { Sr. } \\
\text { No. }\end{array}$ & $\operatorname{Drug}(s)$ & Mobile Phase (v/v) & $\begin{array}{l}\text { Detection } \\
(\lambda \mathbf{n m})\end{array}$ & $\begin{array}{c}\text { Linearity } \\
\mu \mathrm{g} / \mathrm{mL}\end{array}$ & $\begin{array}{c}\mathbf{t R} \\
(\mathrm{min})\end{array}$ & $\begin{array}{c}\text { Correlation } \\
\text { Coefficient }\left(\mathbf{r}^{2}\right) \\
\end{array}$ & Reference \\
\hline 1 & TDF & $\mathrm{H}_{2} \mathrm{O}: \mathrm{MeOH}(45: 55)$ & 230 & $0-120$ & 5.69 & 0.996 & {$[15]$} \\
\hline 2 & TDF & HCCOH:ACN(50:50) & 305 & $5-30$ & 6.718 & 0.9993 & {$[16]$} \\
\hline 3 & TDF & MeOH: ACN: OPA (85:10:05) & 260 & $5-35$ & 2.3 & 0.999 & {$[17]$} \\
\hline 4 & TDF & $\mathrm{NaH}_{2} \mathrm{PO}_{4}$ buffer (pH2.3): $\mathrm{MeOH}(49: 51)$ & 260 & $50-300$ & 9.437 & 0.9995 & {$[18]$} \\
\hline 5 & TDF & $\begin{array}{l}\text { Acetate Buffer pH } 2.8: \text { ACN : } \mathrm{MeOH}(40 \text { : } \\
40: 20)\end{array}$ & 260 & $20-60$ & 2.47 & 0.9992 & {$[19]$} \\
\hline 6 & TDF & ACN: $\mathrm{H}_{2} \mathrm{O}(75: 25)$ & 259 & $0.2-10$ & 1.92 & 0.9966 & {$[20]$} \\
\hline 7 & TDF & $\mathrm{NaH}_{2} \mathrm{PO}_{4}$ buffer (pH 2.3): $\mathrm{MeOH}(49: 51)$ & 260 & $50-300$ & 9.437 & 0.9995. & {$[21]$} \\
\hline 8 & $\begin{array}{l}\text { TDF+ } \\
\text { EMT }\end{array}$ & $\begin{array}{l}\mathrm{ACN}: 50 \mathrm{mM} \mathrm{Na}_{2} \mathrm{HPO}_{4}(\mathrm{pH} 6.0): \text { TEA } \\
(50: 50: 0.1)\end{array}$ & $\begin{array}{c}\text { TDF } \\
260 \\
\text { EMT } \\
280\end{array}$ & $\begin{array}{c}\text { TDF - } \\
0.3-100 \\
\text { EMT } \\
0.8-80\end{array}$ & $\begin{array}{c}\text { TDF } \\
4.98 \\
\text { EMT } \\
5.99\end{array}$ & $\begin{array}{l}0.99996 \\
0.99995\end{array}$ & {$[22]$} \\
\hline
\end{tabular}


$9 \mathrm{TDF}+\mathrm{MeOH}$ : Phosphate buffer(65:35)

EMT

260

(1)

10 TDF+ ACN: $\mathrm{MeOH}: \mathrm{H}_{2} \mathrm{O}(30: 50: 20)$

EMT

11 TDF+ ACN: Phosphate buffer(pH 3.5)(60:40)

EMT

258

27

270

260

12 TDF+ Phosphate Buffer pH 3.0: ACN (60:40)

EMT

260

(1)

13 TDF+ ACN: Phosphate (pH 3.5) buffers (60:40) EMT

270

14

TDF+ ACN: 10 mM Phosphate buffer (pH 6.8)

EMT (60:40)

15 TDF+ ACN: $\mathrm{KH}_{2} \mathrm{PO}_{4}$ buffer ( $\left.\mathrm{pH} 3.0\right):$ TEA

EMT (70:30:0.5)

16 TDF+ ACN: Phosphate buffer (pH 6.8)(60:40)

EMT

17 TDF+ MeOH: ACN : TEA

EMT (46:50:04)

18 TDF+ TEA buffer(pH5.0):ACN:

LMV $\mathrm{MeOH}(30: 40: 30)$

$19 \mathrm{TDF}+\mathrm{KH}_{2} \mathrm{PO}_{4}$ buffer

LMV (pH6.0): $\mathrm{MeOH}: \mathrm{H}_{2} \mathrm{O}(33: 65: 2)$

20 TDF+ ACN: $\mathrm{H}_{2} \mathrm{O}(80: 20)$

LMV

$21 \mathrm{TDF}+\mathrm{MeOH}: \mathrm{H}_{2} \mathrm{O}(85: 15) \mathrm{ACN}:$ Buffer $(\mathrm{pH}$

$\mathrm{EMT}+3.5)(70: 30)$

RPV
260

265

\begin{tabular}{|c|c|c|}
\hline TDF & TDF & 0.999 \\
\hline $10-50$ & 6.231 & \\
\hline EMB & EMB & 0.999 \\
\hline $10-50$ & 2.461 & \\
\hline TDF & TDF & 0.9998 \\
\hline $1-6$ & 2.77 & \\
\hline EMT & EMT & \\
\hline $2-12$ & 3.49 & 0.9999 \\
\hline TDF & TDF & 0.999 \\
\hline $3-15$ & 2.85 & \\
\hline EMT- & EMT & \\
\hline $2-10$ & 3.55 & 0.999 \\
\hline TDF & TDF & 0.999 \\
\hline $75-450$ & 4.5 & \\
\hline EMT- & EMT & \\
\hline $50-300$ & 3.3 & 0.999 \\
\hline TDF- & TDF-2.84 & TDF - \\
\hline $3-15$ & EMT-3.55 & 0.999 \\
\hline EMT- & & EMT- \\
\hline $2-10$ & & 0.999 \\
\hline DF- $60-360$ & TDF-7.42 & TDF- \\
\hline EMT- & EMT-2.81 & 0.999 \\
\hline $40-240$ & & EMT- \\
\hline & & 0.993 \\
\hline TDF- & TDF- 2.27 & TDF- \\
\hline $5-50$ & & 0.9986 \\
\hline EMT- & EMT-1.78 & EMT- \\
\hline $5-50$ & & 0.9995 \\
\hline TDF- & TDF-3.89 & TDF- \\
\hline $6-36$ & EMT-2.883 & 0.999 \\
\hline EMT- & & EMT- \\
\hline $4-24$ & & 0.999 \\
\hline TDF- & TDF- & TDF- \\
\hline $40-100$ & 1.5 & 0.999 \\
\hline EMT- & EMT- 3.5 & EMT- \\
\hline $40-100$ & & 0.999 \\
\hline TDF- & 9.437 & 0.999 \\
\hline $50-300$ & & \\
\hline LMV- & & 0.999 \\
\hline $50-300$ & & \\
\hline TDF- & TDF- & TDF- \\
\hline $50-150$ & 3.4 & 0.9989 \\
\hline LMV- & LMV- & LMV- \\
\hline $50-150$ & 2.3 & 0.9983 \\
\hline $5-25$ & TDF- & TDF- \\
\hline & 2.933 & 0.9987 \\
\hline & LMV-6.966 & LMV- \\
\hline & & 0.9981 \\
\hline TDF- & TDF- & 0.999 \\
\hline $120-1440$ & 8.386 & \\
\hline EMT- & EMT-6.250 & 0.999 \\
\hline $80-960$ & RPV- & \\
\hline RPV- & 10.296 & 1.000 \\
\hline $10-120$ & & \\
\hline
\end{tabular}

[23]

[24]
260

65

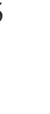




\begin{tabular}{|c|c|c|c|c|c|c|c|}
\hline 22 & $\begin{array}{l}\text { TDF+ } \\
\mathrm{MT}+ \\
\mathrm{NVP}\end{array}$ & $\mathrm{NH}_{4}$ Ac buffer $(\mathrm{pH}-4.6): \mathrm{ACN}$ & 254 & $\begin{array}{c}73.10-219.31 \\
59.76-179.29 \\
120.10-360.30\end{array}$ & $\begin{array}{l}7.89 \\
4.31 \\
6.76\end{array}$ & $\begin{array}{l}1.000 \\
1.000 \\
1.000\end{array}$ & [36] \\
\hline 23 & $\begin{array}{c}\text { TDF+ } \\
\mathrm{LMV+} \\
\text { EFV }\end{array}$ & MeOH:Phosphate buffer $(70: 30)$ & 254 & $\begin{array}{c}\text { TDF- } \\
1-6 \\
\text { LMV- } \\
1-6 \\
\text { EFV- } \\
2-12\end{array}$ & $\begin{array}{c}\text { TDF-3.96 } \\
\text { LMV-2.76 } \\
\text { EFV-10.5 }\end{array}$ & $\begin{array}{l}0.9991 \\
0.9992 \\
0.9995\end{array}$ & {$[37]$} \\
\hline 24 & $\begin{array}{c}\text { TDF+ } \\
\text { EMT+ } \\
\text { EFV }\end{array}$ & $\mathrm{ACN}: \mathrm{H}_{2} \mathrm{O}(60: 40)$ & 260 & $\begin{array}{c}\text { TDF- } \\
6-72 \\
\text { EMT- } \\
4-48 \\
\text { EFV- } \\
12-144\end{array}$ & $\begin{array}{c}\text { TDF-3.860 } \\
\text { EMT-3.105 } \\
\text { EFV- } \\
10.549\end{array}$ & $\begin{array}{c}\text { TDF- } \\
1.0 \\
\text { EMT- } \\
0.999 \\
\text { EFV- } \\
1.0\end{array}$ & [38] \\
\hline 25 & $\begin{array}{c}\text { TDF+ } \\
\text { EMT+ } \\
\text { EFV }\end{array}$ & MeOH: buffer (pH 4.5) & 260 & $\begin{array}{c}\text { TDF- } \\
80-160 \\
\text { EMT- } \\
40-120 \\
\text { EFV- } \\
200-280\end{array}$ & $\begin{array}{l}\text { TDF } \\
\text { EMT } \\
\text { EFV }\end{array}$ & $\begin{array}{c}\text { TDF-0.999 } \\
\text { EMT-0.999 } \\
\text { EFV-0.999 }\end{array}$ & [39] \\
\hline 26 & $\begin{array}{c}\text { TDF+ } \\
\mathrm{LMV+} \\
\text { EFV }\end{array}$ & $\begin{array}{l}\text { phosphate buffer }(\mathrm{pH} 4) \text { : Water: ACN } \\
(30: 70)\end{array}$ & 260 & $\begin{array}{c}\text { TDF- } \\
75-450 \\
\text { LMV- } \\
75-450 \\
\text { EFV- } \\
150-900\end{array}$ & $\begin{array}{c}\text { TDF-2.76 } \\
\text { LMV-3.96 } \\
\text { EFV-10.5 }\end{array}$ & $\begin{array}{c}\text { TDF- } \\
0.9991 \\
\text { LMV- } \\
0.9992 \\
\text { EFV- } \\
0.9995\end{array}$ & {$[40]$} \\
\hline 27 & $\begin{array}{c}\text { TDF+ } \\
\mathrm{LMV+} \\
\mathrm{EFV}\end{array}$ & phosphate buffer (pH 4) : ACN (42:58) & 254 & $\begin{array}{l}\text { TDF- } \\
10-50 \\
\text { LMV- } \\
10-50 \\
\text { EFV - } \\
20-100\end{array}$ & $\begin{array}{c}\text { TDF-3.276 } \\
\text { LMV-2.220 } \\
\text { EFV- } \\
10.814\end{array}$ & $\begin{array}{l}\text { TDF- } \\
0.999 \\
\text { LMV- } \\
0.999 \\
\text { EFV- } \\
0.999\end{array}$ & {$[41]$} \\
\hline
\end{tabular}

Table 2: HPLC chromatographic columns and optimized analytical parameters.

\begin{tabular}{|c|c|c|c|c|c|c|c|}
\hline $\begin{array}{l}\text { Sr. } \\
\text { No }\end{array}$ & Drugs & Column & $\begin{array}{c}\text { Dimensions }(\mathrm{mm}), \\
\operatorname{Particles}(\mu \mathrm{m})\end{array}$ & Detector & $\begin{array}{l}\text { Flow rate } \\
\mathrm{mL} / \mathrm{min}\end{array}$ & $\begin{array}{l}\text { Mode of } \\
\text { analysis }\end{array}$ & References \\
\hline 1 & TDF & Develosil ODS HG -5 RP & $150 \times 4.6,5$ & UV & 1.3 & Isocratic & [15] \\
\hline 2 & TDF & Luna C18 & $250 \times 4.6,5$ & UV & 0.8 & Isocratic & {$[16]$} \\
\hline 3 & TDF & Inertsil ODS C18 & $250 \times 4.6,5$ & UV/visible & 1.0 & Isocratic & {$[17]$} \\
\hline 4 & $\mathrm{TDF}$ & Inertsil ODS-3 & $150 \times 4.6,5$ & PDA & 1.0 & Isocratic & {$[18]$} \\
\hline 5 & TDF & Symmetry C18 & $150 \times 4.6,5$ & UV/Visible & 1.0 & Isocratic & {$[19]$} \\
\hline 6 & TDF & CLC C18 & $25 \times 4.6,5$ & SPD10AVP & 1.0 & Isocratic & {$[20]$} \\
\hline 7 & TDF & Develosil ODS HG -5 RP & $150 \times 4.6,5$ & UV/Visible & 1.3 & Isocratic & {$[21]$} \\
\hline 8 & $\mathrm{TDF}+\mathrm{EMT}$ & Inertsil ODS-3 & $150 \times 4.6,5$ & UV & 1.0 & Isocratic & {$[22]$} \\
\hline 9 & $\mathrm{TDF}+\mathrm{EMT}$ & Zorbax SB-C8 & $250 \times 4.6,5$ & UV & 0.4 & Isocratic & {$[23]$} \\
\hline 10 & $\mathrm{TDF}+\mathrm{EMT}$ & Phenomenax C-18 & $250 \times 4.6,5$ & UV/Visible & 1.0 & Isocratic & {$[24]$} \\
\hline 11 & $\mathrm{TDF}+\mathrm{EMT}$ & Phenomenax Luna C18 & $150 \times 4.6,5$ & UV/visible & 0.6 & Isocratic & {$[25]$} \\
\hline 12 & TDF+EMT & Hypersil C18 & $250 \times 4.6,5$ & PDA & 1.0 & Isocratic & {$[26]$} \\
\hline
\end{tabular}




\begin{tabular}{|c|c|c|c|c|c|c|c|}
\hline 13 & $\mathrm{TDF}+\mathrm{EMT}$ & Phenomenax Luna C18 & $250 \times 4.6,5$ & UV/Visible & 1.0 & Isocratic & {$[27]$} \\
\hline 14 & $\mathrm{TDF}+\mathrm{EMT}$ & Phenomenax Luna C18 & $250 \times 4.6,5$ & UV & 1.0 & Isocratic & [28] \\
\hline 15 & $\mathrm{TDF}+\mathrm{EMT}$ & Luna C18 & $250 \times 4.6,5$ & UV & 1.5 & Isocratic & [29] \\
\hline 16 & TDF+EMT & Luna C18 & $250 \times 4.6,5$ & UV & 1.0 & Isocratic & {$[30]$} \\
\hline 17 & TDF+EMT & Chromosil C-18 & $250 \times 4.6,5$ & UV & $0.5-1.5$ & Isocratic & {$[31]$} \\
\hline 18 & TDF+LMV & Symmetry C8 & $150 \times 4.6,5$ & UV/Visible & 1.0 & Isocratic & {$[32]$} \\
\hline 19 & TDF+LMV & Thermosil C18 & $150 \times 4.6,3.5$ & UV & 0.8 & Isocratic & {$[33]$} \\
\hline 20 & TDF+LMV & $\mathrm{RP}-\mathrm{C} 18$ & $250 \times 4.6,5$ & UV & 0.7 & Gradient & [34] \\
\hline 21 & $\begin{array}{c}\mathrm{TDF}+\mathrm{EMT} \\
+\mathrm{RPV}\end{array}$ & Inertsil ODS 3V & $250 \times 4.6,5$ & PDA & 1.5 & Gradient & [35] \\
\hline 22 & $\begin{array}{c}\mathrm{TDF}+\mathrm{EMT} \\
+\mathrm{NVP}\end{array}$ & Inertsil ODS-2 & $150 \times 4.6,5$ & $\mathrm{DAD}$ & 1.0 & Isocratic & [36] \\
\hline 23 & $\begin{array}{c}\mathrm{TDF}+\mathrm{LMV} \\
+\mathrm{EFV}\end{array}$ & Kromasil C18 & $150 \times 4.6,5$ & UV & 1.0 & Isocratic & {$[37]$} \\
\hline 24 & $\begin{array}{c}\text { TDF+EMT } \\
+ \text { EFV }\end{array}$ & Hypersil BDS C18 & $250 \times 4.6,5$ & PDA & 0.8 & Isocratic & [38] \\
\hline 25 & $\begin{array}{c}\text { TDF+EMT- } \\
\text { EFV }\end{array}$ & Zorbax SB CN & $250 \times 4.6,5$ & UV & 1.5 & Isocratic & [39] \\
\hline 26 & $\begin{array}{c}\mathrm{TDF}+\mathrm{LMV} \\
+\mathrm{EFV}\end{array}$ & Hypersil C18 & $150 \times 4.6,5$ & PDA & 1.0 & Gradient & {$[40]$} \\
\hline 27 & $\begin{array}{c}\mathrm{TDF}+\mathrm{LMV} \\
+\mathrm{EFV}\end{array}$ & Symmetry C18 & $100 \times 4.6,3.5$ & UV & 0.5 & Gradient & [41] \\
\hline
\end{tabular}

Table 3: Pharmaceutical Analysis of TDF/TNF in biological fluids.

\begin{tabular}{|c|c|c|c|c|c|c|c|}
\hline $\begin{array}{l}\text { Sr. } \\
\text { No }\end{array}$ & $\operatorname{Drug}(s)$ & $\begin{array}{l}\text { Sample } \\
\text { matrix }\end{array}$ & Method & $\begin{array}{c}\text { Column } \\
\text { Dimensions }(\mathrm{mm}), \\
\text { particle }(\mu \mathrm{m})\end{array}$ & Detector & Internal standard & References \\
\hline 1 & TNF & $\begin{array}{l}\text { Human } \\
\text { plasma }\end{array}$ & LC/MS & $\begin{array}{ll}\mathrm{RP} & \text { Chromolith, } \\
\mathrm{C} 18,100 \times 4.6,5\end{array}$ & $\begin{array}{l}\text { UV, fluorescence, } \\
\text { and LC-tandem MS }\end{array}$ & Adefovir & {$[42]$} \\
\hline 2 & $\mathrm{TNF}$ & $\begin{array}{l}\text { Human } \\
\text { plasma }\end{array}$ & LCMS/MS & $\begin{array}{l}\text { Kromasil100, C18, } 150 \\
\times 4.6,5\end{array}$ & HPLC /UV & Fluconazole & {$[43]$} \\
\hline 3 & TNF & $\begin{array}{l}\text { Human } \\
\text { plasma }\end{array}$ & LC-ESI MS-MS & Supelco C8, $150 \times 4.6,5$ & UV & $\begin{array}{l}\text { Tenofovir and } \\
\text { Tenofovir D6 }\end{array}$ & {$[44]$} \\
\hline 4 & TNF & $\begin{array}{l}\text { Human } \\
\text { plasma }\end{array}$ & LC-MS/MS & $\begin{array}{l}\text { Zorbax } 5 \text { micron SCX } \\
\text { HPLC column, } 3.0 \times 50\end{array}$ & $\begin{array}{l}\text { UV, fluorescence } \\
\text { and MS }\end{array}$ & $\begin{array}{l}\text { Tenofovir and } \\
\text { Tenofovir D6 }\end{array}$ & {$[45]$} \\
\hline 5 & $\mathrm{TNF}+\mathrm{EMT}$ & $\begin{array}{l}\text { Human } \\
\text { plasma }\end{array}$ & LC-MS/MS & $\begin{array}{l}\text { Synergi Polar-RP, } 150 \times \\
2.0 \mathrm{~mm} \text {, }\end{array}$ & MS/MS, ESI & $\begin{array}{l}\text { Iso-TFV and Iso- } \\
\text { FTC }\end{array}$ & {$[46]$} \\
\hline 6 & $\mathrm{TNF}+\mathrm{EMT}$ & $\begin{array}{l}\text { Human } \\
\text { plasma }\end{array}$ & LC-MS/MS & $\begin{array}{l}\text { Synergi Polar RP } \\
\text { HPLC Column pore } \\
\text { size, } 80^{\circ} \mathrm{A}, 150 \times 2.0,4\end{array}$ & HPLC /UV & $\begin{array}{l}\text { TDF- adefovir and } \\
\text { dideoxyuridine } \\
\text { EMT-deoxy- } \\
\text { fluorocytidine }\end{array}$ & {$[47]$} \\
\hline 7 & $\mathrm{TNF}+\mathrm{LMV}$ & $\begin{array}{l}\text { Human } \\
\text { plasma }\end{array}$ & LC-MS/MS & $\begin{array}{l}\text { Chromolith ROD speed } \\
\text { C18 } 250 \times 4.6,5\end{array}$ & HPLC/MS & Abacavir & {$[48]$} \\
\hline 8 & $\mathrm{TNF}+\mathrm{EMT}$ & $\begin{array}{l}\text { Human } \\
\text { plasma }\end{array}$ & LC-MS/MS & $\begin{array}{l}\text { HiQ Sil C18HS } 250 \times \\
4.6,5\end{array}$ & UV & Clonazepam & {$[49]$} \\
\hline
\end{tabular}




\begin{tabular}{cccclccc}
9 & TNF+ EMT & $\begin{array}{c}\text { Human } \\
\text { plasma }\end{array}$ & LC-MS/MS & $\begin{array}{l}\text { Chromolith Speed Rod } \\
\text { RP18 } 50 \times 4.6,5\end{array}$ & MS & Lamivudine & {$[50]$} \\
10 & TNF+ NVP+ & $\begin{array}{c}\text { Human } \\
\text { plasma }\end{array}$ & LC-MS/MS & $\begin{array}{l}\text { Phenomenex kinetex } \\
\text { LMV }\end{array}$ & MS $18,100 \times 4.6,2.6$ & Emtricitabine & [51] \\
\hline
\end{tabular}

\subsubsection{Bioanalytical methods of analysis}

Total ten bioanalytical methods are reported so far for determination of TNF in biological samples either individual or in combination with other antiretroviral drugs viz EMT, LMV and NVP (Yadav et al., 2009; Rao et al., 2013; Paliwal et al., 2014; Podany et al., 2015; Delahunty et al., 2009; Zheng et al., 2014; Matta et al., 2012; Patel et al., 2015; Gomes et al., 2008; Valluru et al., 2013). In most of these methods extraction of TNF and other drugs in its combination from human plasma have been performed using solid-phase extraction , liquid-liquid extraction, protein precipitation and solid -phase analytical derivatization were applied. Solvents used for extraction of TNF and other drugs from biological matrix include acetonitrile, methanol, water, trichloroacetic acid, and trifluoroacetic acid etc. The internal standards viz Adefovir, fluconazole, Tenofovir, Tenofovir D6, Abacavir, Clonazepam, dideoxyuridine and deoxy-fluorocytidine were used during analysis. The separation of the TNF and other drugs were performed on different analytical column such as Chromolith Chromolith ROD speed C18, Kromasil100, Synergi PolarRP Discovery Supelco C8, Phenomenex kinetex C18, HiQSil C18HS, Zorbax 5 micron SCX HPLC and Prontosil C18AQ with dimension $(100 / 150 / 250 \times 2 / 4.6 \mathrm{~mm}, 5 \mu \mathrm{m})$ under isocratic mode of analysis was used. Various proportion of mobile phase containing combination of acetonitrile, methanol and water was applied. Wherever necessary the $\mathrm{pH}$ of the water was adjusted using formic acid/ ammonium acetate/ ammonium formate as modifier. In most of these analytical methods, the detection of TNF was performed by applying MS/ ESI -MS/MS/Tandem- MS/ Fluorescence/UV detectors. Details about, sample matrices, method type, $\lambda$-max, column, and use of internal standard if any are shown in Table 3.

\subsubsection{HPTLC/TLC - densitometry determinations}

Five HPTLC methods have been reported for TDF alone and for simultaneous estimation of TDF in combination with other drugs such as EMT and RPV (Pradeep et al., 2011; Mardia et al., 2012; Joshi et al., 2009; Rao et al., 2011; Saminathan and Vetrichelvan, 2016). The separation carried out on aluminium plates precoated with silica gel using various mobile phase composition such as methanol, chloroform, toluene and most of acidic modifier used for efficient separation such as Ethyl acetate, glacial acetic acid with various proportion. Detection carried out at different wavelength like $265 \mathrm{~nm}, 266 \mathrm{~nm}, 270 \mathrm{~nm}$ and $272 \mathrm{~nm}$. The amount of the TDF and its combined drug estimated in pharmaceutical formulation was reported to be in superior conformity with label claimed. Details of mobile phase composition, detection of $\lambda \max$, linearity, and retention factor and coefficient correlation are shown in Table 4.

Table 4: HPTLC methods for determination of TDF/TNF.

\begin{tabular}{|c|c|c|c|c|c|c|c|}
\hline $\begin{array}{l}\text { Sr. } \\
\text { No }\end{array}$ & $\begin{array}{l}\text { Name of } \\
\text { Drug }\end{array}$ & $\begin{array}{l}\text { Mobile phase composition } \\
\qquad(\mathrm{v} / \mathrm{v})\end{array}$ & $\begin{array}{c}\text { Detection } \\
(\lambda \mathbf{n m})\end{array}$ & $\begin{array}{l}\text { Linearity } \\
\text { (ng/spot) }\end{array}$ & $\mathbf{R}_{\mathrm{f}}$ & $\mathbf{r}^{2}$ & References \\
\hline 1 & $\mathrm{TDF}$ & $\mathrm{CHCl}_{3}: \mathrm{MeOH}(8.5: 1.5)$ & 270 & $200-1200$ & 0.54 & 0.9994 & {$[52]$} \\
\hline 2 & TDF & $\begin{array}{c}\text { EtOAc: } \mathrm{MeOH}: \mathrm{HCOOH} \\
(7: 2.5: 0.5)\end{array}$ & 266 & $125-750$ & 0.78 & 0.991 & {$[53]$} \\
\hline 3 & $\mathrm{TDF}+\mathrm{EMT}$ & $\mathrm{CHCl}_{3}: \mathrm{MeOH}(9: 1)$ & 265 & $200-1000$ & $\begin{array}{l}0.47 \\
0.18\end{array}$ & $\begin{array}{l}0.9996 \\
0.9995\end{array}$ & {$[54]$} \\
\hline 4 & TDF+EMT & $\begin{array}{c}\text { Toluene: } \\
\mathrm{MeOH}: \text { EtOAc: } \mathrm{AcOH} \\
(4: 2: 5: 0.1)\end{array}$ & 270 & $\begin{array}{c}120-600 \\
80-560\end{array}$ & $\begin{array}{l}0.52 \\
0.40\end{array}$ & $\begin{array}{c}0.999 \\
0.9992\end{array}$ & {$[55]$} \\
\hline 5 & $\begin{array}{c}\mathrm{TDF}+ \\
\mathrm{EMT}+\mathrm{RPV}\end{array}$ & $\begin{array}{c}\mathrm{CHCl}_{3}: \text { EtOAc: } \mathrm{MeOH}: \\
\text { Glacial Acetic acid } \\
(5: 2: 1: 0.1)\end{array}$ & 272 & $\begin{array}{c}\text { TDF-600-3600 } \\
\text { EMT- 600-2400 } \\
\text { RPV- 50-300 }\end{array}$ & $\begin{array}{l}\text { TDF- } 0.52 \\
\text { EMT- } 0.28 \\
\text { RPV- } 0.70\end{array}$ & $\begin{array}{l}\text { TDF-0.9993 } \\
\text { EMT- } 0.9992 \\
\text { RPV- } 0.9995\end{array}$ & {$[56]$} \\
\hline
\end{tabular}

\subsubsection{Stability-indicating chromatographic methods}

Seven stability-indicating methods have been found so far for estimation of TDF in bulk and pharmaceuticals using different analytical techniques such as under different prescribed stress conditions (hydrolysis, oxidation, dry and wet heat and photolysis). Out of these, two methods are for reported for estimation of TNF alone and five describe stability-indicating methods for TDF in combination 
EMT, RPV, LMV, NVP, EFV, ELV and COBI (Havele and Dhaneshwar, 2012; Hussen et al., 2013; Agashe et al., 2015; Prasad et al., 2012; Sudha and Manjeera, 2012; Rao et al., 2016; Rao et al., 2014). The details about reported stabilityindicating methods for TDF giving emphasis on sample matrix, mobile phase, $\lambda \max$, linearity, range, retention time and correlation coefficient is shown in Table 5.

Table 5: Stability-indicating methods for analysis of TDF/TNF.

\subsubsection{Spectrophotometric analysis}

About sixteen UV-Spectrophotometry procedures have been studied for determination of TDF alone and in combined dosage forms. Table 6 depicts about spectrophotometry methods with respect to sample matrix, technique, solvent used, $\lambda \max$, linearity range, and correlation coefficient.

\begin{tabular}{|c|c|c|c|c|c|c|c|}
\hline $\begin{array}{l}\text { Sr. } \\
\text { No }\end{array}$ & $\begin{array}{c}\text { Name of } \\
\operatorname{drug}(s)\end{array}$ & $\begin{array}{c}\text { Mobile phase } \\
\operatorname{composition}(\mathbf{v} / \mathbf{v})\end{array}$ & $\begin{array}{c}\text { Detection } \\
(\lambda \mathbf{n m})\end{array}$ & $\begin{array}{c}\text { Linearity } \\
\mu \mathrm{g} / \mathrm{ml}\end{array}$ & $\begin{array}{c}t_{R} \\
(\min )\end{array}$ & $\mathbf{r}^{2}$ & References \\
\hline 1 & TDF & $\mathrm{MeOH}: \mathrm{H}_{2} \mathrm{O}(60: 40)$ & 260 & $4-20$ & 12.09 & 0.9999 & [57] \\
\hline 2 & TDF & $\begin{array}{l}\text { ACN: } 0.025 \mathrm{M} \mathrm{H}_{2} \mathrm{PO}_{4} \text { buffer } \\
(\mathrm{pH} 3.0)(35: 65)\end{array}$ & 260 & $0.1-50$ & 7.5 & 0.999 & {$[58]$} \\
\hline 3 & $\mathrm{TDF}+\mathrm{EMT}$ & $\begin{array}{l}\mathrm{NH}_{4} \mathrm{Ac} \text { buffer }(\mathrm{pH} \quad 5.5): \\
\mathrm{MeOH}(85: 15)\end{array}$ & 260 & $\begin{array}{l}\text { TDF-10-60 } \\
\text { EMT-10-60 }\end{array}$ & $\begin{array}{l}\text { TDF-31.83 } \\
\text { EMT-11.6 }\end{array}$ & $\begin{array}{l}\text { TDF- } 0.9991 \\
\text { EMT-0.999 }\end{array}$ & [59] \\
\hline 4 & $\begin{array}{c}\text { TDF+ } \\
\mathrm{LMV+} \\
\mathrm{NVP}\end{array}$ & $\begin{array}{l}\text { A. }\left(\mathrm{NH}_{4}\right)_{3} \mathrm{PO}_{4}: \text { dil. TFA } \\
\text { B. } \mathrm{MeOH}: \text { ACN. }(10: 90)\end{array}$ & 260 & $0.05-0.50$ & $\begin{array}{l}\text { TDF-38 } \\
\text { LMV-11 } \\
\text { NVP-27 }\end{array}$ & $\begin{array}{l}\text { TDF-0.997 } \\
\text { LMV-0.998 } \\
\text { NVP-0.999 }\end{array}$ & {$[60]$} \\
\hline 5 & $\begin{array}{c}\text { TDF+ } \\
\mathrm{LMV}+\mathrm{EFV}\end{array}$ & $\begin{array}{l}\text { TEA buffer: } \mathrm{MeOH} \\
(35: 65)\end{array}$ & 260 & $\begin{array}{l}\text { TDF-75-225 } \\
\text { LMV-75 -225 } \\
\text { EFV-150-450 }\end{array}$ & $\begin{array}{l}\text { TDF-3.36 } \\
\text { LMV-2.43 } \\
\text { EFV-10.46 }\end{array}$ & $\begin{array}{l}\text { TDF-0.999 } \\
\text { LMV-0.999 } \\
\text { EFV-0.999 }\end{array}$ & {$[61]$} \\
\hline 6 & $\begin{array}{c}\mathrm{TDF}+ \\
\mathrm{EMT}+\mathrm{EFV}\end{array}$ & $\begin{array}{l}\mathrm{MeOH}: \text { TEA } \\
(70: 30)(\mathrm{pH} 7)\end{array}$ & 260 & $\begin{array}{l}\text { TDF-15-75 } \\
\text { EMT-10-50 } \\
\text { EFV-10-50 }\end{array}$ & $\begin{array}{c}\text { TDF-4.632 } \\
\text { EMT-3.706 } \\
\text { EFV-8.121 }\end{array}$ & $\begin{array}{l}\text { TDF- } 0.999 \\
\text { EMT- } 0.999 \\
\text { EFV- } 0.999\end{array}$ & {$[62]$} \\
\hline 7 & $\begin{array}{c}\text { TDF+ } \\
\text { EMT+ } \\
\text { COBI+ } \\
\text { ELV }\end{array}$ & 0.1\%TFA: ACN & 242 & $\begin{array}{c}\text { TDF-150-450. } \\
\text { EMT-100-300 } \\
\text { COBI-75-225 } \\
\text { ELV-75-225 }\end{array}$ & $\begin{array}{c}\text { TDF- } 4.75 \\
\text { EMT- } 3.43 \\
\text { COBI- } 5.27 \\
\text { ELV- } 7.56\end{array}$ & $\begin{array}{l}\text { TDF- } 0.999 \\
\text { EMT-0.999 } \\
\text { COBI-0.999 } \\
\text { ELV- } 0.999\end{array}$ & {$[63]$} \\
\hline
\end{tabular}

Table 6: Spectrophotometric methods used for determination of TDF alone and in combined dosage form.

\begin{tabular}{|c|c|c|c|c|c|c|c|}
\hline $\begin{array}{l}\text { Sr. } \\
\text { No }\end{array}$ & Name of drug & & / order & $\begin{array}{c}\text { Detection } \\
(\lambda, \mathbf{n m})\end{array}$ & $\begin{array}{c}\text { Linearity } \\
(\mu \mathrm{g} / \mathrm{ml})\end{array}$ & $\begin{array}{c}\left(\mathbf{r}^{2}\right) \\
\text { Value }\end{array}$ & References \\
\hline 1 & TDF & I & Zero & 451 & $8-40$ & 0.998 & [64] \\
\hline 2 & TDF & I & Zero & 261 & $5-90$ & 0.9983 & {$[65]$} \\
\hline 3 & TDF & $\begin{array}{l}\text { I } \\
\text { II }\end{array}$ & $\begin{array}{l}\text { Zero } \\
\text { AUC }\end{array}$ & $\begin{array}{c}260 \\
250-270\end{array}$ & $5-30$ & $\begin{array}{l}0.999 \\
0.999\end{array}$ & [66] \\
\hline 4 & TDF & I & Zero & 259 & $5-45$ & 0.9995 & {$[67]$} \\
\hline 5 & TDF & $\begin{array}{l}\text { I } \\
\text { II }\end{array}$ & $\begin{array}{l}\text { Zero } \\
\text { AUC }\end{array}$ & $\begin{array}{c}260 \\
250-270\end{array}$ & $5-25$ & $\begin{array}{l}0.992 \\
.9917\end{array}$ & [68] \\
\hline 6 & TDF & $\begin{array}{l}\text { I } \\
\text { II }\end{array}$ & $\begin{array}{l}\text { Zero } \\
\text { First }\end{array}$ & $\begin{array}{l}260 \\
273\end{array}$ & $4-24$ & $\begin{array}{l}0.999 \\
0.998\end{array}$ & [69] \\
\hline 7 & TDF & I & Zero & 260 & $10-50$ & 1.00004 & {$[70]$} \\
\hline 8 & $\mathrm{TDF}+\mathrm{EMT}$ & I & $\mathrm{ACM}$ & $\begin{array}{l}\text { TDF-260 } \\
\text { EMT-290 }\end{array}$ & $\begin{array}{l}\text { TDF- 5-25 } \\
\text { EMT-7-35 }\end{array}$ & $\begin{array}{l}0.999 \\
0.999\end{array}$ & [71] \\
\hline 9 & $\mathrm{TDF}+\mathrm{EMT}$ & I & Zero & $\begin{array}{l}\text { TDF-240 } \\
\text { EMT-280 }\end{array}$ & $10-80$ & $\begin{array}{l}0.9996 \\
0.9993\end{array}$ & [72] \\
\hline
\end{tabular}


$\mathrm{TDF}+\mathrm{EMT}$

$\begin{array}{cc}\text { I } & \text { First } \\ \text { II } & \text { Derivative } \\ \text { III } & \text { ACM }\end{array}$

11

14

15

ACM

SEM

SEM

$$
\mathrm{TDF}+\mathrm{EMT}
$$

I

$\begin{array}{cccc}13 & \text { TDF+LMV } & \text { I } & \text { SEM } \\ 14 & \text { TDF+LMV } & \text { I } & \text { SEM } \\ 15 & \text { TDF+ EMT+ RPV } & \text { I } & \text { SEM }\end{array}$

EMT-210, 281

\begin{abstract}
TDF-306.88
EMT-224.38

EMT-293.38

TDF-270.29
\end{abstract}

TDF-260

EMT- 290

TDF-210, 281
TNF-271
LAM-261
TDF- 260
LMV-280
TNF-259
EMT-280
RPV-291

$\begin{array}{cc}\text { TDF-3-21 } & \text { TDF- } \\ \text { EMT-2-14 } & 0.9999 \\ \text { EMT-4-20 } & 0.9996 \\ \text { TDF- 6-30 } & 0.9997 \\ \text { TDF- 5-25 } & 0.999 \\ \text { EMT- 7-35 } & 0.999 \\ 4-24 & 0.9997 \\ & 0.99977 \\ & 0.99978 \\ & 0.99991 \\ 5-50 & 0.999 \\ & 0.999 \\ \text { TDF- 5-45 } & 0.999 \\ \text { LMV-2-16 } & 0.998 \\ \text { TNF-3-21 } & 0.9993 \\ \text { EMT-1-10 } & 1.000 \\ \text { RPV-0.5-3 } & 0.9996\end{array}$

[73]

\subsubsection{Spectrophotometry analysis as a single drug}

Three different methods have been reported for determination of TDF in tablet dosage form as a single component. All these methods reported for analysis implemented zero order, first order and Area under curve (AUC) techniques. Water was used as a solvent and analysis were carried out at appropriate wavelength (Buridi, 2013; Gnanarajan et al., 2013; Balaji et al., 2012; Mondal and Singh, 2014; Srujani et al., 2015; Himaja et al., 2014; Rani et al., 2012)

\subsubsection{Simultaneous spectrophotometry determinations in various dosage forms}

TDF is available with many antiviral drugs in combined tablet dosage form. The range of UV-spectrophotometry method for simultaneous determination of TNF in the pharmaceutical formulation has been established. Five different methods established for determination of TDF in tablets as multiple components have been reported. All these methods studied for analysis of TNF employed Zero order, First order, Derivative method, ACM and SEM methods using water and methanol as a solvent. The combination of TDF, EMT, LMV and RPV has been estimated using ICH guidelines. (Viswanath et al., 2013; Sasikala et al., 2013; Choudhari et al., 2011; Srinivasan et al., 2014; Ananda et al., 2011; Dubbaka et al., 2015; Soumya et al., 2012; Madhuri et al., 2014)

\subsection{A Typical Methods of Pharmaceutical Importance}

A simple and reliable method of capillary electrophoresis (CE) combined with field-amplified sample stacking, using hydroxypropyl methyl cellulose as electro-osmotic flow suppressant has been reported for separation and identification of TNF and Adefovir, using phosphate buffer solution containing $0.3 \%$ of hydroxypropyl methylcellulose and measured at $18 \mathrm{kV}$ or $214 \mathrm{~nm}$. The method has been successfully established for determination of TNF and Adefovir in bovine serum and which is suitable for pharmacokinetic study. The prominent characteristic as claimed by author is to be highly sensitive, cost-effective, relatively simple, and time-saving than other methods such as LC/MS/MS, HPLCUV, Fluorescent derivation HPLC, and SPME-HPLC (Liu and Duan 2015). The electrochemical reduction of (TNF) was studied in Britton-Robinson (BR) buffer at the dropping mercury drop electrode (HMDE). A method based on square-wave cathodic adsorptive stripping voltammetry (SWCAdSV) was studied and validated for assay of TNF in human plasma and a tablet formulation. Sample preparation of plasma involved protein precipitation with acetonitrile. The method reported to be linear in the concentration range $0.5-5.0 \mu \mathrm{g} / \mathrm{ml}$. The method was reported to quite inexpensive compare to other methods (Jain and Sharma 2013).

Quantification of TDF in pharmaceutical formulations via chemical derivatization using p-chloranilic acid and sodium nitroprusside as a reagent have been studied as extractions free spectrophotometric methods determination of TDF in bulk drugs and pharmaceutical formulations (tablets). The first method reported to be based upon the charge transfer complexation reaction between drug as anelectron donor and $p$-chloranilic acid ( $\mathrm{p}-\mathrm{CA}$ ) as a $\mathrm{p}$-acceptor to form a violet chromogen measured at $531 \mathrm{~nm}$. The colored product depicts linearity in the concentration range of 2-10 $\mu \mathrm{g} / \mathrm{mL}$. The second method was reported to be based on the formation of light green molecular complex with sodium nitroprusside in presence of hydroxylamine under alkaline 
conditions and exhibiting $\lambda$-max at $401 \mathrm{~nm}$ and showed linearity in theconcentration range of $2-10 \mu \mathrm{g} / \mathrm{ml}$ (Disha and Gurupadayya 2013). A solid-phase extraction (SPE) method has been studied and validated on a liquid chromatography coupled with a mass spectrometer for the determination of plasma concentrations of TDF and EMT in HIV patients. Separation was achieved with a gradient (Acetonitrile and water with formic acid $0.05 \%$ ) on an Atlantis $4.6 \mathrm{~mm} \times 150$ $\mathrm{mm}$, reversed-phase analytical column. Detection of TNF, EMT, and internal standard (IS) was achieved by electrospray ionization mass spectrometry (ESI-MS) in the positive ion mode. Plasma was analyzed, and the limit of quantitation was $15.6 \mathrm{ng} / \mathrm{mL}$ for TNF and $11.7 \mathrm{ng} / \mathrm{mL}$ for EMT; limit of detection was $2 \mathrm{ng} / \mathrm{mL}$ for TNF and $1.5 \mathrm{ng} /$ $\mathrm{mL}$ for EMT. Mean recovery of TNF, EMT, and IS were $46.5 \%$ [relative standard deviation (RSD): $8.8 \%$ ] and 88.8 \% (RSD: $1.0 \%$ ), and $81.7 \%$ (RSD: $3.1 \%$ ), respectively. The method was applied for the estimation of drug plasma concentration of HIV patients treated with EMT and TNF, in combination with others (D'Avolio et al., 2008).

\section{Conclusion}

Review of literature reported during the period of 2008 -2016 described analytical methods for estimation of TDF. Different analytical methods implemented for analysis of TDF in bulk and in its combined dosage forms and in plasma include LC-MS/MS, HPLC, HPTLC and UVSpectroscopy methods. Fig. 2 depicts method prevalence for pharmaceutical analysis of TDF and Fig. 3 give comparative account on various methods published using different analytical techniques for TDF.

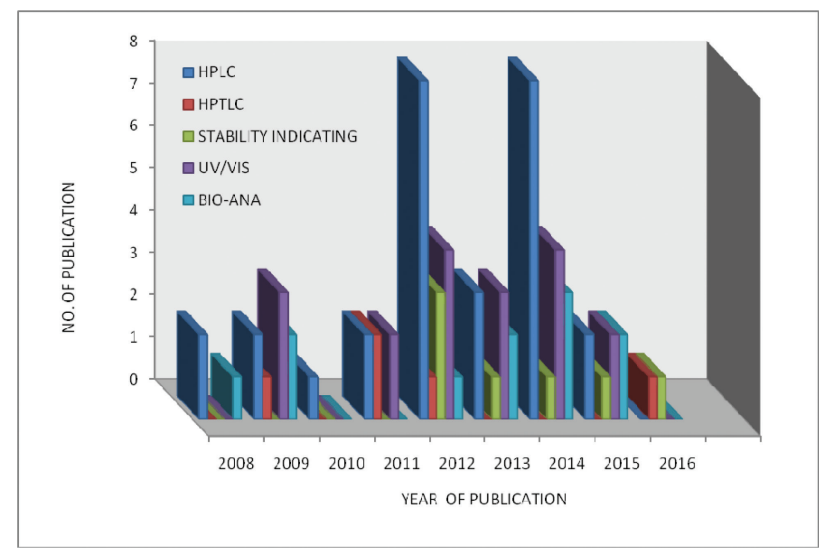

Figure 2: Method Prevalence for Pharmaceutical Determination of TDF.

One of the most commonly used solvents for sample extraction of TDF from pharmaceutical formulation and also from biological fluids is methanol and acetonitrile.
The detection of HPLC analysis of most of methods was monitored at $260 \mathrm{~nm}$ with PDA/UV detector. The mobile phase composition for the separation of TDF includes methanol, acetonitrile and buffer or their combination in appropriate proportion. The present review article can provide wisdom to the reader about the various methods employed for analysis of TDF and update them with several options for analysis of TDF. The date compiled may be useful for the further analysis of TDF.

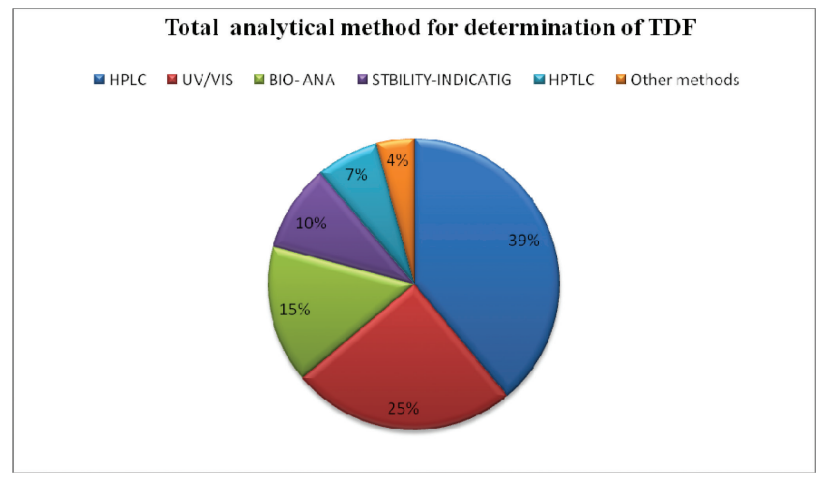

Figure 3: Total Analytical Methods for determination of TDF

\section{Acknowledgement}

Authors are grateful to R. C. Patel Institute of Pharmaceutical Education and Research Shirpur, (MS) for providing necessary library facilities.
Abbreviations Used
- ACM- Absorbance correction method
- ADV-Adefovir
- API- Active Pharmaceutical ingredient
- AUC- Area under curve
- COBI- Cobicistat
- EFV- Efavirenz
- ELV- Elvitegravir
- EMT- Emtricitabine
- HBV-Hepatitis B virus
- HIV-Human immune- deficiency virus
- HMDE- Hanging mercury drop electrode
- HPLC- High performance liquid chromatography
- HPTLC-High performance thin layer chromatography
- LC- Liquid chromatography
- LMV-Lamivudine
- M.P-Melting point
- $\mathrm{Na}_{2} \mathrm{HPO}_{4}$-Disodium phosphate
- NVP- Nevirapine
- OPA- o- phosphoric acid 
- p-CA- p-chloranilic acid

- $\mathrm{pH}$ - power of hydrogen

- RF- Retention factor

- RPV- Rilpivirine

- RT- Retention time

- SEM-Simultaneous equation method

- $\quad$ SIM- stability indicating method

- $\quad$ SPME- solid phase microextraction method

- TDF-Tenofovir Disofroxil fumarate

- TEA- Triethylamine

- TNF- Tenofovir

\section{References}

1. Goicoechea M., Liu S., Best B., Sun S., Jain S., Kemper C., Witt M., Diamond C., Haubrich R. and Louie S. (2008). Greater tenofovir-associated renal function decline with protease inhibitor-based versus nonnucleoside reverse-transcriptase inhibitor-based therapy, J. Infectious Diseases, 197(1), 102-108. https://doi.org/10.1086/524061

2. Avihingsanon A., Sophonphan J., Thammajaruk N., Chaihong P., Burger D., Cressey T. R., Ramautarsing R. A., Praditornsilpa K., Avihingsanon Y., Ruxrungtham K. and HIV-NAT 114 study team. (2015). Plasma Tenofovir Concentrations and Proximal Tubular Dysfunction in HIV-Infected Adults Receiving Tenofovir in Thailand, J. AIDS \& Clinical Research, 6(7), 477.

https://doi.org/10.4172/2155-6113.1000477

3. Patel K. K., Patel A. K., Ranjan R. R., Patel A. R. and Patel J. K. (2010). Tenofovir-associated renal dysfunction in clinical practice: An observational cohort from western India, Indian J. STD and AIDS, 31(1), 30-34.

https://doi.org/10.4103/0253-7184.68998

4. Bygrave H., Ford N., van C. G., Hilderbrand K., Jouquet G., Goemaere E., Vlahakis N., Trivino L., Makakole L. and Kranzer K. (2011). Implementing a tenofovir-based first-line regimen in rural Lesotho: clinical outcomes and toxicities after two years, J. AIDS, 56(3), e75-e78. https://doi.org/10.1097/QAI.0b013e3182097505

5. The Merck Index, 13 TH Editions, Merck Research Laboratories, Merck and CO., White House Station, NJ, USA, 2001, 1631-1632.

6. Kearney B. P., Flaherty J. F. and Shah J.: Tenofovir disoproxil fumarate, Clinical Pharmacokinetics, 2004, 43(9), 595-612.

https://doi.org/10.2165/00003088-20044309000003

7. James C. W., Steinhaus M. C., Szabo S. and Dressler R. M. (2004). Tenofovir-related nephrotoxicity: case report and review of the literature. Pharmacotherapy, The Journal of Human Pharmacology and Drug Therapy, 24(3), 415-418. https://doi.org/10.1592/phco.24.4.415.33182

8. www.Gilead.com/pdf/viread_pi.pdf; Gilead Science Inc. Viread, US prescribing information.

9. Birkus G., Hitchcock M. J. and Cihlar T. (2002). Assessment of mitochondrial toxicity in human cells treated with tenofovir: comparison with other nucleoside reverse transcriptase inhibitors, Antimicrobial Agents and Chemotherapy, 46(3), 716-723. https://doi.org/10.1128/AAC.46.3.716-723.2002

10. Cihlar T., Birkus G., Greenwalt D. E. and Hitchcock M. J. (2002). Tenofovir exhibits low cytotoxicity in various human cell types: comparison with other nucleoside reverse transcriptase inhibitors, Antiviral research, 54(1), 37-45.

https://doi.org/10.1016/S0166-3542(01)00210-8

11. Porche D. J. (2002). Tenofovir Disoproxil Fumarate $\left(\operatorname{Viread}^{\mathrm{TN}}\right)$, Journal of the Association of Nurses in AIDS Care, 13(3), 100-102.

https://doi.org/10.1177/10529002013003007

12. Fontana R. J. (2009). Side effects of long-term oral antiviral therapy for hepatitis B, Hepatology, 49(S5), S185-S195. https://doi.org/10.1002/hep.22885

13. Rajput J. K., Patil P. H., Surana S. J. and Shirkhedkar A. A. (2015). Analytical Methods for Determination of Muscle Relax-ant Thiocolchicoside in Pharmaceutical Preparations-A Review, Open Pharmaceutical Sciences Journal, 2(1), 43-55. https://doi.org/10.2174/1874844901502010043

14. Siddiqui M. R., AlOthman Z. A. and Rahman N. (2013). Analytical techniques in pharmaceutical analysis: A review, Arabian Journal of chemistry, 2-46.

15. Sarala K., Subrahmanyam K. V. and Patnaik A. (2014). Method Development and Validation of Tenofovir In Its Bulk Form by Using RP-HPLC, International Journal of Medicine and Nanotechnology, 1(3), 122-126.

16. SM M. and Nandedkar M. A. (2009). Determination of Tenofovir Disoproxil Fumarate by a Sensitive Simple Isocratic RP-HPLC Method, J. Ind. Council Chem, 26(1), 67-69.

17. Sundar B. S. and Edla S. (2011). Rp-HPLC Method Development and Validation for the Analysis of Tenofovuir in Pharmaceutical Dosage Forms, Journal of Atoms and Molecules, 1(1), 32-40.

18. Sharma T., Mishra N., Moitra S. S. and Sankar D. G. (2012). A validated RP-HPLC method for estimation of Tenofovir disoproxil fumarate in bulk and pharmaceutical formulation, Asian Journal of Pharmaceutical and Clinical Research, 5(3), 108-110. 
19. Balaji M., Srikanth I., Reddy S, H., Krishna G. R., Ramu S., Kumar V. A. (2012). Development and Validationof RP-HPLC Methodfor Quantitative Estimation of Tenofovir Disproxil Fumarate in Bulk and Pharmaceutical Dosage Form. Journal of Pharmacy Research, 5(9), 4692-4694.

20. Kandagal P. B., Manjunatha D. H., Seetharamappa J. and Kalanur S. S. (2008). RP-HPLC Method for the Determination of Tenofovir in Pharmaceutical Formulations and Spiked Human Plasma, Analytical Letters, 41(4), 561-570. https://doi.org/10.1080/00032710801910742

21. Sharma T., Mishra N., Moitra S. S. and Sankar D. G. (2010). A validated RP-HPLC method for estimation of Tenofovir disoproxil fumarate in bulk and pharmaceutical formulation, Asian Journal of Pharmaceutical and Clinical Research, 5(3), 108-110.

22. Abdelhay M., Gazy A., Shaalan R. and Ashour H. (2013). Selective RP-HPLC-DAD method for determination of tenofovir fumarate and emtricitabine in bulk powder and in tablets, Acta Chromatographica, 27(1), 41-54.

https://doi.org/10.1556/AChrom.27.2015.1.4

23. Lavanya B., Hariprasad P., Venkatapraveen A., Lakshmi D. P. and Ramireddy M. (2012). Simultaneous estimation of emtricitabine and tenofovir disproxilfumarate by HPLC method, Pharmacia Lettre. Scholars Research Library, 4(6), 1855-1862.

24. Karunakaran A., Kamarajan K. and Thangarasu V. (2010). A validated RP-HPLC method for simultaneous estimation of emtricitabine and tenofovir disoproxil Fumarate in pure and in tablet dosage form, Pharm Sin, Pelagia Research Library, 1(2), 52-60.

25. Dr. Srinivasan R., Mary K. L., Lakshmana G., Kumar D. R., Rajini B. (2014). Method development and validation of tenofovir disoprpxilfumarate and emtricitabine in combined tablet dosage form by UVspectrophotometry and RP-HPLC, Int. J. of Pharmacy and Analytical Research, 3(4), 414-421.

26. Venkatesh M., Sumanth A. L., M. N., Rao P. V. (2013). Analytical Method Development And Validation Of Simultaneous Estimation Of Tenofovir And Emtricitabine In Bulk And Pharmaceutical Dosageforms By Using RP-HPLC, Asian Journal of Pharmaceutical Analysis and Medicinal Chemistry, 1(2), 60-69.

27. Viswanath V., Shanmugasundaram P., Ravichandiran V. (2013). RP-HPLC Method For The Simultaneous Estimation of Tenofovir Disoproxil Fumarate And Emtricitabine in Combined Tablet Dosage Form, International Journal of PharmTech Research, 5(3), 1186-1195.
28. Yenumula B. R., Singampalli M, R. and Challa, B, S., (2015). Simultaneous Estimation of Emtricitabine and Tenofovir Disoproxil Fumarate in Tablet Dosage Form by Reverse Phase High-performance Liquid Chromatography. SOJ Chromatographic Science. 1(1), pp.2-6. https://doi.org/10.15226/2471-3627/1/1/00104

29. Sharma, R. and Gupta, P., (2009). Simultaneous Quantification and Validation of Emtricitabine and Tenofovir Disoproxil Fumarate in a Tablet Dosage Form. Eurasian Journal of Analytical Chemistry, 4(3), 276-284.

30. Vanitha C., Kumar P. P., Sekhar R. V., Swarnalatha G. and Sekar V. (2014). Simultaneous Estimation Of Emtricitabine And Tenofovir Disoproxil Fumarate In A Tablet Dosage Form By RP-HPLC Method, International Journal of Pharmaceutical Development \& Technology, 4(4), 279-283.

31. Devanaboyina N., Satyanarayana T. and Rao G. (2012). HPLC method development and validation for simultaneous estimation of tenofovir and emtricitabine in combined pharmaceutical dosage form, International Journal of Research in Pharmaceutical and Biomedical Sciences, 3(1), 361-367.

32. Kumar A. K., Chaitanya K. K. and Babu N. S. (2012). Development And Validation of Reverse Phase HPLC Method For Determination of Lamivudine and Tenofovir in Binary Mixture, Journal of Global Trends in Pharmaceutical Sciences, 3(3), 849-852.

33. Gorja A. and Bandla, J. (2011). Method development and validation for the simultaneous estimation of Lamivudine and Tenofovir disoproxil fumarate in pharmaceutical dosage forms by RP-HPLC, International Journal of Advanced Research in Pharmaceutical and Biomedical Sciences, 2(1), 22-32.

34. Mali A. S., Salunke P. A., Barhate S. D. and Bari M. M. (2015). Simultaneous Determination of Lamivudine and Tenofovir Disproxil Fumarate by UV Spectrophotometric Method, Word journal of Pharmaceutical Research, 4(3), 995-1006.

35. Khan A., Rao V. J., Pulla R. P., Kumar S. S. and Sujana K. (2014). Simultaneous Estimation of Emtricitabine, Tenofovir Disoproxil Fumarate And Rilpivirine $\mathrm{Hcl}$ In Tablet Dosage Forms By Rp-HPLC, International Journal of Pharmaceutical Research \& Analysis, 4(1), 23-30.

36. Jayapalu K., Malipeddi H. and Chinnasamy A. (2014). Chromatographic Separation and in Vitro Dissolution Assessment of Tenofovir disoproxil fumarate, Emtricitabine and Nevirapine in a Fixed 
Dose Combination of Antiretrovirals, Journal of Applied Pharmaceutical Science, (11), 076-080.

37. Bhavsar D. S., Patel B. N. and Patel C. N. (2012). RPHPLC method for simultaneous estimation of tenofovir disoproxil fumarate, lamivudine, and efavirenz in combined tablet dosage form, Pharmaceutical methods, 3(2), 73-78. https://doi.org/10.4103/2229-4708.103876

38. Raju N. A., Rao J. V., Prakash K. V., Mukkanti K. and Srinivasu K. (2008). Simultaneous estimation of tenofovir disoproxil, emtricitabine and efavirenz in tablet dosage form by RPHPLC, Oriental Journal of Chemistry, 24(2), 645-650.

39. Ramaswamy A. and Dhas A. S. A. G.: Development and validation of analytical method for quantitation of Emtricitabine, Tenofovir, Efavirenz-based on HPLC, Arabian Journal of Chemistry, 2014.

40. Prathap B., Dey A. and Rao G. H. S. (2014). Analytical Method Development And Validation Of Invitro Dissolution Studies Of Efavirenz, Lamivudine And Tenofovir Disoproxil Fumarate In Pharmaceutical Dosage Form By RP-HPLC, Journal of Global Trends in Pharmaceutical Sciences, 5(2), $515-520$

41. Bhargavi G. T., Mohammad Y. R. and Reddy Y. (2012). Method Development and Validation of Lamivudine, Tenofovir and Efavirenz in a Combined Dosage Form by RP-HPLC, Journal of Pharmacy Research, 5(12), 711-714.

42. Yadav M., Mishra T., Singhal P., Goswami S. and Shrivastav P. S. (2009). Rapid and specific liquid chromatographic-tandem mass spectrometric determination of tenofovir in human plasma and its fragmentation study, Journal of Chromatographic Science, 47(2), 140-148.

https://doi.org/10.1093/chromsci/47.2.140

43. Rao N., Bathula V. And Devi K. G. (2013). Method Development And Method Validation of Tenofovir In Human K3 Edta Plasma By Lc-Ms/Ms, Pharma Science Monitor, 4(3), 73-76.

44. Paliwal N., Jain P., Dubey N., Sharma S., Khurana S. and Paliwal S. K. (2014). A liquid chromatography tandem mass spectrometry-based regulatory compliant method for the determination of tenofovir in human serum, Drug research, 64(06), 306-312.

45. Podany A. T., Sheldon C., Grafelman D. and Ohnmacht C. M. (2015). Assay development for determination of tenofovir in human plasma by solid-phase analytical derivatization and LC-MS/MS, Bioanalysis, 7(24), 3085-3095. https://doi.org/10.4155/bio.15.220
46. Delahunty T., Bushman L., Robbins B. and Fletcher C. V. (2009). The simultaneous assay of tenofovir and emtricitabine in plasma using LC/MS/MS and isotopically labeled internal standards, Journal of Chromatography B, 877(20), 1907-1914. https://doi.org/10.1016/j.jchromb.2009.05.029

47. Zheng J. H., Guida L. A., Rower C., CastilloMancilla J., Meditz A., Klein B., Kerr B. J., Langness J., Bushman L., Kiser J. and Anderson P. L. (2014). Quantitation of tenofovir and emtricitabine in dried blood spots (DBS) with LC-MS/MS, Journal of pharmaceutical and biomedical analysis, 88, 144-151. https://doi.org/10.1016/j.jpba.2013.08.033

48. Matta M. K., Burugula L., Pilli N. R., Inamadugu J. K. and Jvln S. R. (2012). A novel LC-MS/MS method for simultaneous quantification of tenofovir and lamivudine in human plasma and its application to a pharmacokinetic study, Biomedical Chromatography, 26(10), 1202-1209. https://doi.org/10.1002/bmc.2679

49. Patel A., Patel A. And Makwana A. (2015). Development And Validation Of An Accurate And Precise Lc-Ms/Ms Method for Determination of Tenofovir And Emtricitabine in Human Plasma, International Journal Of Pharmaceutical, Chemical \& Biological Sciences, 5(1), 372-380.

50. Gomes N. A., Vaidya V. V., Pudage A., Joshi S. S. and Parekh S. A. (2008). Liquid chromatographytandem mass spectrometry (LC-MS/MS) method for simultaneous determination of tenofovir and emtricitabine in human plasma and its application to a bioequivalence study, Journal of Pharmaceutical and Biomedical Analysis, 48(3), 918-926. https://doi.org/10.1016/j.jpba.2008.07.022

51. Valluru R. K., Kumar P. and Kilaru N. B. (2013). High throughput LC-MS/MS method for simultaneous determination of tenofovir, lamivudine and nevirapine in human plasma, Journal of Chromatography B, 931, $117-126$.

https://doi.org/10.1016/j.jchromb.2013.05.008

52. Pradeep K., Dwivedi S. C. and Ashok K. (2011). Validated HPTLC method for the determination of tenofovir as abulk drug and in pharmaceutical dosage form, Res J Chem Sci, 1(7), 33-37.

53. Mardia R. B., Suhagia B. N., Pasha T. Y., Chauhan S. P. and Solanki S. (2012). Development and Validation of HPTLC Method for Estimation of Tenofovir Disoproxil Fumarate in Tablet Dosage Form, Journal of pharmaceutical science and bioscientific research, 2(2), 73-76.

54. Joshi M., Nikalje A. P., Shahed M. and Dehghan M. (2009). HPTLC method for the simultaneous estimation of emtricitabine and tenofovir in tablet 
dosage form, Indian Journal of Pharmaceutical Sciences, 71(1), 95-97. https://doi.org/10.4103/0250-474X.51951

55. Rao J., Gondkar S. and Yadav S. (2011). Simultaneous HPTLC-Densitometric analysis of Tenofovir and Emtricitabine in Tablet dosage form, Int J Pharm Tech Res, 3(3), 1430-1434.

56. Saminathan J. and Vetrichelvan T. (2016). Development and Validation of HPTLC Method for Simultaneous Estimation of Emtricitabine, Rilpivirine and Tenofovir Disoproxil. Fumarate in Combined Dosage form, Bangladesh Pharmaceutical Journal, 19(1), 114-121.

https://doi.org/10.3329/bpj.v19i1.29247

57. Havele S. and Dhaneshwar S. R. (2012). Development and validation of a stability-indicating lc method for the determination of tenofovir disoproxil fumarate in pharmaceutical formulation, Songklanakarin Journal of Science and Technology, 34(6), 612-622.

58. Hussen S. S., Shenoy P. and Krishna M. (2013). Development and validation of stability indicating RPHPLC method for tenofovir nanoparticle formulation, Int. J. Pharm. Pharm. Sci, 5(2), 245-248.

59. Agashe M., Chaudhari R., Shirsat V. (2015). A Rapid Stability Indicating RP-HPLC Method and the Degradation Kinetics Data for the Simultaneous Estimation of Tenofovir Disoproxil Fumarate and Emtricitabine, Human Journals Research Article, 3(3), 145-159.

60. Prasad L. A., Rao J. V., Shrinivasu P., Prasad J. V. and Pavani G. (2012). New Stability Indicatingrp-Lc Method For Simultaneous Quantification Of Related Impurities Of Lamivudine, Tenofovir Disoproxil Fumarate And Nevirapine In Extended Release Tablet Dosage Form, Int Research Journals Pharmacy, 3(11), 103-107.

61. Sudha T. and Manjeera K. K. (2012). Stability indicating RP-HPLC method for the simultaneous estimation of the anti-retroviral drugs and in tablet dosage forms, International Journal of Biology, Pharmacy and Allied Sciences, 1(9), 1322-1335.

62. Rao S., Kumar N., Srilekha K. And Kumari A. (2016). Stability Indicating Method For The Simultaneous Estimation Of Tenofovir, Emtricitabine And Efavirenz In Pure And Pharmaceutical Dosage Form By RPHPLC, International Journal of Advance Research In Science And Engineering, 5(5), 188-200.

63. Rao P. P., Reddy D. M. and D. Ramachandran (2014). Stability indicating HPLC method for simultaneous estimation of emtricitabine, tenofovir disoproxyl fumarate, cobicistat and elvitegravir in pharmaceutical dosage form, World Journal of Pharmaceutical Sciences, 2(12), 1822-1829.

64. Buridi K. R. (2013). Quantitative Determination of Tenofovirdisproxil Fumarate as an Active PharmaceuticalIngredient in Tablet Formulations by Visible Spectrophotometry, Asian Journal of Pharmaceutical Research and Health Care, 5(1), 24-31.

65. Gnanarajan G., Gupta A., Juyal V., Kumar P., Yadav P. and Kailash P. (2009). A validated method for development of tenofovir as API and tablet dosage forms by UV spectroscopy, Journal of Young Pharmacists, 1(4), 351-353.

https://doi.org/10.4103/0975-1483.59326

66. Balaji M., Moorthy G., Perumal R. V., Sumanth K. S., Channabasavaraj K. P. (2012). Development And Validation of UV spectrophotometric Methods for Quantitative Estimation of Tenofovir Disproxil Fumarate in Bulk \& Pharmaceutical Dosage Form, International Journal of Pharmaceutical Research \& Analysis, 2(1), 14-17.

67. Mondal N. And Singh Y. (2014). Development and Validation of Different Spectrophotometric Methods for Estimation of Tenofovir Disoproxil Fumarate From Bulk Drug and Tablets, International Journal of Pharmaceutical Sciences And Research, 5(2), 623-629.

68. Srujani C., Sowmya D. K., Murthy V. S. and Rani A. P. (2015). Development and validation of stability indicating aUVspectrophotometric method for the estimation of tenofovir in its bulk and pharmaceutical dosage form, Pharmacia Lettre, Scholars Research Library, 7(9), 177-185.

69. Himaja M., Kalpana J. and Anbarasu C. (2014). Validated zero order and first order derivative spectrophotometric methods for in-vitro analysis of Tenofovir disoproxil fumarate tablets using azeotropic mixture, Int J Pharm Pharm Sci, 6(6), 302-304.

70. Rani T. S., Sujatha K., Chitra K., Jacob D., Yandapalli R., Manoj D. and Susma B.: Spectrophotometric methods for estimation of tenofovir disoproxilfumarate in tablet, Res Rev J Pharm Anal, 2012, 1(1), 9-12.

71. Viswanath V., Shanmugasundaram P., Ravichandiran V. (2013). UV-Spectrophotometric Absorbance Correction Method for the Simultaneous Estimation of Tenofovir Disoproxil Fumarate and Emtricitabine in Combined Tablet Dosage Form, International Journal of PharmTech Research, 5(3), 1179-1185.

72. Sasikala M., Deepthi M. T., Kumar K. V., Raja N. M. (2013). Development And Validation of UV Spectrophotometric Method For The Simultaneous Estimation of Tenofovir Disproxil Fumarate And 
Emtricitabine In Tablet Dosage Form By Derivative Spectroscopic Method, International Bulletin of Drug Research, 3(5), 20-28.

73. Choudhari V. P., Ingale S., Gite S. R., Tajane D. D., Modak V. G. and Ambedkar A. (2011). Spectrophotometric simultaneous determination of Tenofovir disoproxil fumarate and Emtricitabine in combined tablet dosage form by ratio derivative, first order derivative and absorbance corrected methods and its application to dissolution study, Pharmaceutical methods, 2(1), 47-52. https://doi.org/10.4103/2229-4708.81096

74. Dr. Srinivasan R., Mary K. L., Lakshmana G., Kumar D. R., Rajini B. (2014). Method development and validation of tenofovir disoprpxilfumarate and emtricitabine in combinedtablet dosage form by UVspectrophotometry and RP-HPLC. Int. J. of Pharmacy and Analytical Research, 3(4), 414-421.

75. Ananda kumar K., Kannan K. and Vetrichelvan T. (2011). Development and validation of emtricitabine and tenofovir disoproxilfumarate in pure and in fixed dose combination by UV Spectrophotometry, Digest Journal of Nanomaterials and Biostructures, 6(3), 1085-1090.

76. Dubbaka A., Sireesha D., Basha S. A., Talla R., Haque M. A., Harshini S., Vasudha B. (2015). Analytical Method Development and Validation forthe Simultaneous Estimation of Lamivudine and Tenofovir Disoproxil Fumarate in Bulk and Spectrophotometric Method, International Journal of Innovative Pharmaceutical Sciences and Research, 3(10), 1529-1536.

77. Soumya B., Kumar T. M. and Raghunandhan N. (2012). Simultaneous determination of Tenofovir disoproxil fumarate and Lamivudine by UV
Spectrophotometric Method, International Journal of Pharmacy and Pharmaceutical Science Research, 2(1), 9-15.

78. Madhuri M., Sri K. V. And Jain G. V. (2014). Simultaneous Estimation And Validation of Tenofovir Disproxil Fumarate, Emtricitabine And Rilpivirine Hydrochloride In Pharmaceutical Dosage Form By UV-Spectrophotometry, Indo-American Journal of Pharmaceutical Research, 4(12), 5864-5873.

79. Liu J. and Duan Y. (2015). Field-Amplified on-line Sample Stacking for Separation and Determination of Adefovir and Tenofovir Using Capillary Electrophoresis, Asian Journal of Chemistry, 27(9), 3143. https://doi.org/10.14233/ajchem.2015.15412

80. Jain R. and Sharma, R. (2013). Cathodic adsorptive stripping voltammetric detection and quantification of the antiretroviral drug tenofovir in human Plasma and a tablet Formulation, Journal of The Electrochemical Society, 160(8), H489-H493. https://doi.org/10.1149/2.105308jes

81. Disha N. S. And Gurupadayya B. M. (2013). Determination of Tenofovir Disoproxil Fumarate an Pharmaceutical Formulations By P-Chloranilic Acid and Sodium Nitroprusside as Reagent. International Journal of Advances in Pharmaceutical Research. 4(9), 2189-2195.

82. D'Avolio A., Sciandra M., Siccardi M., Baietto L., de Requena D. G., Bonora S. and Di Perri G. (2008). A new assay based on solid-phase extraction procedure with LC-MS to measure plasmatic concentrations of tenofovir and emtricitabine in HIV-infected patients, Journal of chromatographic science, 46(6), 524-528. https://doi.org/10.1093/chromsci/46.6.524 